NISTIR 7909

\title{
Toward an Integrated Decision Support Framework for Sustainability Analysis
}

Lalit Patil

Lakshmi Srinivas

Krishna Murthy

Debasish Dutta

Rachuri Sudarsan

http://dx.doi.org/10.6028/NIST.IR.7909

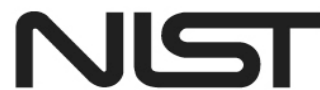

National Institute of Standards and Technology U.S. Department of Commerce 
NISTIR 7909

\title{
Toward an Integrated Decision Support Framework for Sustainability Analysis
}

\author{
Lalit Patil \\ Lakshmi Srinivas \\ Krishna Murthy \\ BIMCON Inc. \\ Debashish Dutta \\ University of Illinois at Urbana-Champaign \\ Rachuri Sudarsan \\ Systems Integration Division \\ Engineering Laboratory
}

http://dx.doi.org/10.6028/NIST.IR.7909

January 2013

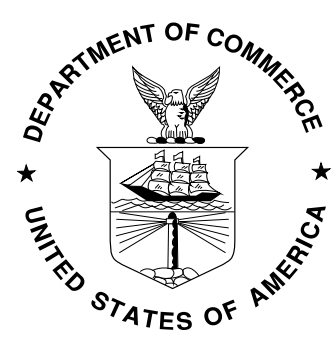

U.S. Department of Commerce Rebecca Blank, Acting Secretary

National Institute of Standards and Technology Patrick D. Gallagher, Under Secretary of Commerce for Standards and Technology and Director 


\title{
Toward an Integrated Decision Support Framework for Sustainability Analysis
}

\author{
Lalit Patil $^{1}$, Lakshmi Srinivas ${ }^{2}$, Krishna Murthy ${ }^{3}$, Debasish Dutta ${ }^{4}$, and Rachuri Sudarsan ${ }^{5}$
}

\begin{abstract}
Sustainability evaluation is typically conducted after the product is completely designed. However, sustainability, like cost and weight, needs to be managed on a continuous basis, throughout the product development cycle. This requires a decision support tool that provides sustainability analyses estimates at early design phases to guide detailed design. Such a decision support tool can be facilitated only through the complete integration of sustainability data into product development, in particular with Computer Aided Engineering (CAE) tools, methods, and processes. We present work toward developing an effective decision support framework for the CAE-level analysis of sustainability using information models that integrate sustainability data across design and engineering analysis tools, especially within the target management framework of the automotive industry. This framework is called Sustainability CAE (SCAE). In particular, we present an information model to capture the main components of knowledge required in CAE processes with interfaces to using various sustainability metrics. We establish the feasibility of this framework through the design, implementation, and analysis of a proof of concept that captures a typical design scenario in the automotive industry. We conclude with a discussion on potential topics for further research in this area.
\end{abstract}

Keywords: Sustainable manufacturing, Information modeling, Sustainability CAE, Target management

\section{Background}

Computer Aided Engineering (CAE) refers to the computer-based analysis of a product's performance in different conditions. Such studies are carried out for various purposes, e.g., improving designs, and help in avoiding the need to build prototypes. The objective of the work presented in this paper is to establish the feasibility of a decision support framework that transforms the current time-consuming and reactive (post completion of the final design) sustainability analysis into a proactive CAE-level approach. This approach should enable design revisions based on performance against sustainability targets during the design process, i.e., enable design optimization for sustainability. This framework, called Sustainability CAE (SCAE) will integrate the latest advancements in open standards and software capabilities to: (a) capture lifecycle-wide information relevant to sustainability and its assessment, and (b) organize and integrate it in a usable form for analysis. Much of the insights and applications in this paper are relevant to the automotive industry, and we use a "vehicle” as a representative product throughout this paper.

\footnotetext{
${ }^{1}$ Lalit Patil is with BIMCON Inc., W. Bloomfield, MI 48323 USA and the Mechanical Science and Engineering Department, University of Illinois at Urbana-Champaign, Urbana, IL 61801 (e-mail: lpatil@bimcon.com).

${ }^{2}$ Lakshmi Srinivas is an independent consultant with BIMCON Inc., W. Bloomfield, MI 48323 USA (e-mail: ylsrinivas@yahoo.com).

${ }_{3}^{3}$ Krishna Murthy is with BIMCON Inc., W. Bloomfield, MI 48323 USA (e-mail: kmurthy@bimcon.com).

${ }^{4}$ Debasish Dutta is with the Mechanical Science and Engineering Department, University of Illinois at UrbanaChampaign, Urbana, IL 61801 (e-mail: ddutta@illinois.edu).

${ }^{5}$ Rachuri Sudarsan is with the National Institute of Standards and Technology, Gaithersburg, MD 20899 (e-mail: rachuri.sudarsan@nist.gov).
} 


\subsection{Sustainable manufacturing}

Sustainability is now firmly established as a top priority for companies and governments around the world, and, in particular, in the United States. Sustainable manufacturing is defined as a "systems approach for the creation and distribution of innovative products and services that minimizes resources, eliminates toxic substances, and produces zero waste" [1]. In the face of global changes in the consumer tastes and preferences, Original Equipment Manufacturers (OEMs) view sustainable manufacturing as a mandate for competitiveness. As the Aberdeen group suggests, "by optimizing their green product development programs across the entire development lifecycle, manufacturers can reduce costs, meet product launch dates and drive new business" [2]. Aligned with this view, the U.S. federal government, through the American Recovery and Reinvestment Act of 2009 [3] calls for investments in "environmental protection and other infrastructure that will provide long-term economic benefits." Its goal is to provide opportunities for the US industry to demonstrate leadership in sustainable manufacturing, such as in zero carbon footprint (CF) and 100 percent recyclability through complete disassembly of the product.

Sustainability requirements may apply to the entire product or portions of the product. Modern consumer products are complex assemblies of components some of which are built in-house by the OEM and others are procured from suppliers. However, it is the OEM's responsibility to ensure that the product and its components conform to all relevant requirements. This calls for effective evaluation of sustainability throughout the supply chain. Furthermore, integrating such evaluation into the product development cycle will enhance the OEM's ability to manage sustainability along the various phases in the cycle.

Incorporating a lifecycle-wide framework into product development to enable sustainable manufacturing is a major challenge facing manufacturing companies today. Companies face problems in accessing key information to meet sustainability goals and there is a lack of effective tools that are expected to address important challenges, like informed decision-making, policy integration, and stakeholder participation [4].

To enable effective analysis of sustainability, in particular environmental impact, the International Organization for Standardization (ISO) has specified principles and a generic framework for life cycle assessment (LCA). Currently, LCA is widely used for assessing the environmental impact of products and services [5]. It is a methodology that considers several aspects from across the lifecycle and their environmental impact. A commercial example of assessment of sustainability is that of Ford Motor Company's Product Sustainability Index that covers 8 different components of sustainability that include safety, emissions, and lifecycle costs [6]. Most of the current LCA approaches to assess sustainability are post-design and cannot be used proactively during the design process.

Note that a few sustainability elements, such as safety and emissions, driven by regulatory requirements, are implemented very well in automotive product development. These are supported with robust analysis tools and techniques. Weight is a surrogate for a number of sustainability metrics like lifecycle costs and fuel economy; it is also actively managed on most product programs. Yet, there exists no "sustainability attribute" ${ }^{\prime 1}$ on any vehicle program, and hence sustainability does not get the visibility or management exposure it needs to be fully effective as a driver for product development and manufacture.

\footnotetext{
${ }^{1}$ An Attribute is a specific customer/corporate/regulation-driven parameter the product behavior. This interpretation is different from the typical interpretation in data modeling, where the terms, attribute, property, and parameter are usually used interchangeably.
} 
It is known that the management of an attribute, as a driver for product development, requires that it be in an enterprise's target management framework, in which targets can be established for the attribute. In this framework, the attribute has the capabilities to be assessed and its status published periodically. In addition, some tools are available for executives to act in accordance with corporate/regulatory requirements. Thus, for sustainability to be a driver for product development, it must be an attribute and part of a target management framework. This is discussed in the next section.

\subsection{Current state of sustainability evaluation/assessment}

Complexity of the products - a typical automotive vehicle program has more than 250,000 lines in its Bill of Material (BOM) - limits the ability of companies to fully validate sustainability or other performance measures through physical testing. Therefore, virtual/analytical assessments are increasingly used to fill the gap for effective design decisions and optimization. As CAE techniques and hardware capabilities continuously improve, the ability of OEMs to analytically prove-out their designs also grows. Such analysis procedures, which are now an integral part of the product development process, focus primarily on assessing functional performance, such as stress analysis on components and assemblies using finite element methods, thermal and fluid flow analysis, kinematics, and vehicle noise analysis.

Typically, weight and cost are managed quite effectively as product attributes in in design and analysis. However, the inclusion of sustainability metrics in design and analysis is sporadic and inconsistent. Vehicle safety is an example of a sustainability metric that is included in design and analysis.

We believe that, like cost and weight, sustainability as a whole needs to be managed as a product attribute, on a continuous basis, throughout the product development cycle. This calls for design aids that can facilitate quick and accurate calculation and assessment of the metrics, along with the ability to revise designs based on performance against selected sustainability targets in the product design process. This can be facilitated only through a framework that enables a complete integration of sustainability data into the product development toolset in general and in CAE tools, methods, and processes, in particular.

The configuration of a new product model, along with the properties, materials, and processing of the components is decided in early design (conceptual and embodiment design) phases. Therefore, while the abovementioned post-design evaluation is necessary, to truly understand and design products for sustainability, techniques to assimilate lifecycle-wide information into product design tools and facilitate decision-making are required. There is a need for an integrated decision support solution for sustainable manufacturing that enables a proactive approach through which sustainability is embedded into the early product design phases. This corresponds to the need for a solution to enable target management, including evaluation of sustainability metrics, i.e., sustainability requirements are decomposed and assigned to the subsystems as targets; the subsystems are evaluated and if targets cannot be met, corresponding negotiation and rebalancing can be undertaken prior to the completion of the final design.

Incorporating sustainability attributes as targets to be managed, as discussed above, forms a fundamental step toward enabling sustainability as a primary driver for product development, i.e., enabling the vision for Product Sustainability Management (PSM), which can be realized as described in Section 1.4. 


\subsection{Target management framework for sustainability}

Our broad vision is a comprehensive decision support solution for managing and incorporating sustainability in the early phases of product design through the target management framework. To do this, the solution must support the following three processes (See Figure 1):

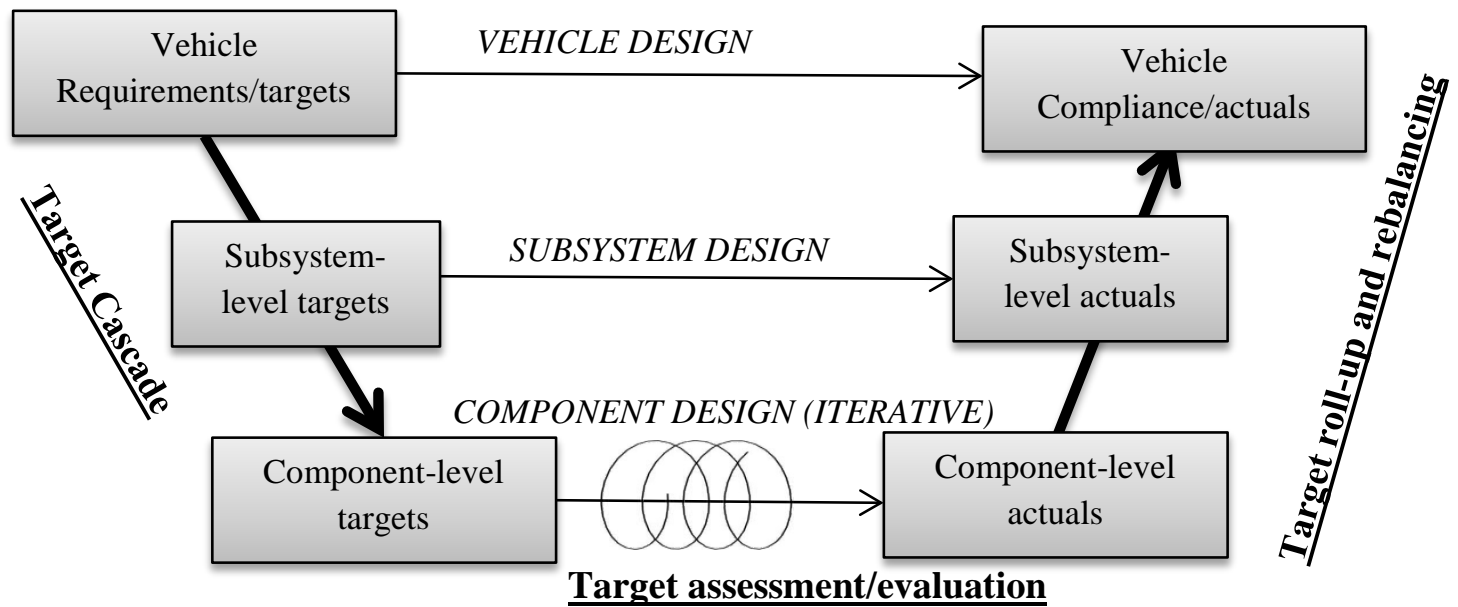

Figure 1: Target cascading, assessment, and roll-up and rebalancing are three main components in incorporating sustainability into early product design. The work documented in this paper focused on target assessment, in particular, on incorporating sustainability during the CAE for component-level evaluations. This work focuses on completing the loop in a timely fashion, i.e., moving from after-the-design component evaluations to in-design system-level evaluations.

1. Sustainability target cascading: In this process, the vehicle-level targets are decomposed into subsystem and component-level targets that design engineers must meet. The values are set in line with the corporate, business and regulatory needs prior to the start of the product development process. The cumulative impact of the component-level targets on the subsystem/vehicle targets will ensure compliance both from engineering and business viewpoints. We address this aspect of target management in a separate project [7].

2. Sustainability target assessment: The actual values of the attributes associated with the components and subsystems are predicted or obtained using various techniques. A few, such as costs, can be, and are, directly assessed. Others, such as vehicle safety, are computed using models and analytical/physical representations. The work reported here has focused on this step, i.e., target assessment, in particular, on incorporating CAE into the sustainability target assessment, i.e., on component level target evaluation.

3. Sustainability target roll-up and rebalancing: Actual values from the assessment are rolled up, i.e., summed up from component to system levels, to verify compliance with the targets. In the case of deviations, rebalancing, i.e., relaxing some and tightening other attribute values to ensure systemlevel compliance, might be needed and newer targets are computed depending on the severity of the deviation. A group of human experts undertakes this rebalancing in the context of all vehicle attributes concurrently. The knowledge embedded in the target cascading, assessment, and results from target roll ups is an important resource that human experts use in tradeoff negotiations. Once newer targets are setup, target cascading is iterated.

The significance of effectively managing and evaluating sustainability targets and the interrelationships among different attributes is evident from the recent changes to the Ford Explorer. The program was about to be abandoned because the excessive weight (2018.5 kg) of the vehicle significantly reduced its 
fuel efficiency. Instead, a stringent target was assigned: designers were required to manage a $45.4 \mathrm{~kg}$ reduction in vehicle weight. The new target was cascaded throughout the vehicle assembly. Successful redesign has led to $24 \%$ increase in fuel efficiency [8] and ensured the vehicle's continuation in the market.

Next, we discuss the current state of sustainability assessment/ evaluation which forms the focus of this paper.

\subsection{The vision of Product Sustainability Management (PSM)}

We envision a framework of PSM, which will ensure that products will be designed holistically with overall sustainability as a primary goal, rather than sustainability being mostly evaluated after the fact. We believe that this framework can be achieved by efforts along the following three phases (shown in Figure 2):

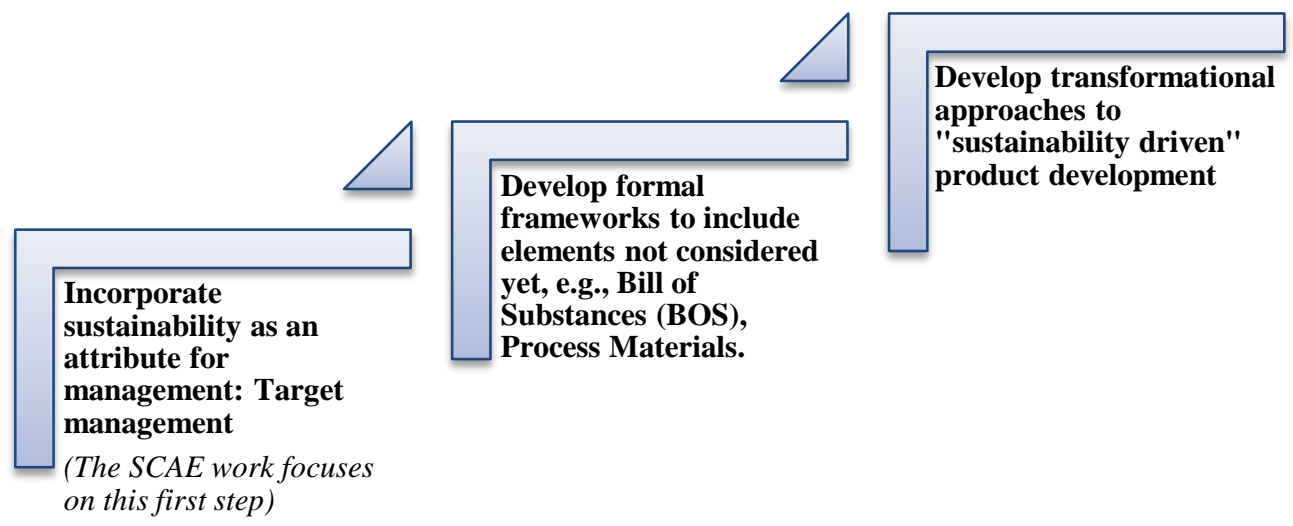

Figure 2: Three fundamental steps toward realizing the broad vision of PSM

1. Incorporating sustainability into the cohort of attributes that are being currently managed effectively by companies. This requires the management of sustainability, as a whole, as a product attribute throughout the product development cycle. This can be achieved by incorporating sustainability in the target management framework, i.e., ensure target cascading, target evaluation, and target rebalancing, as discussed earlier. Our ongoing work on Sustainability Integrated into Early Design (SIED) focuses on target cascading [7]. The SCAE work described in this paper focuses on timely evaluation of sustainability attributes and closing the loop in making the target evaluation for effective tradeoff negotiations. Thus, the SIED work focuses on target cascading (See Figure 1) and the SCAE work focuses on target evaluation, which is done after the targets are cascaded.

2. Developing formal structures and new frameworks for mapping elements that are not effectively managed or even considered in the current Engineering BOM-centric paradigm. Examples of such elements include Bill of Substances and Process Material knowledge. Currently, manufacturing process information, including "process materials" that are used or consumed during the manufacturing is rarely available to the designer. This is very significant for the evaluation of sustainability, since these "process materials" might be significant contributors to the overall sustainability.

3. Developing tools and techniques for a paradigm shift from the current design "cost-driven" BOM-centric development to a "sustainability driven" Sustainable-BOM-centric development. In this paradigm, sustainability, instead of cost, will become the primary driver for product lifecycle 
management. Note that sustainability includes lifecycle cost. This will require the development of transformational methods and techniques based on the foundations mentioned in points (1) and (2) above. It also requires cultural and infrastructural shifts within enterprises.

Sustainability, like cost and weight, needs to be managed on a continuous basis, throughout the product development cycle. This requires a decision support tool that provides sustainability analyses results at early design phases to guide detailed design. This can be facilitated only through the complete integration of sustainability data into product development, in particular with the CAE tools, methods, and processes.

In this work, we demonstrate the feasibility of assessing targets at component-levels. Thus, the goal of our work can be stated as follows:

\section{Development of an effective decision support framework for CAE-level analysis of sustainability using information models that integrate sustainability data across design and engineering analysis tools}

It should be noted that typical evaluation of the status of targets, especially at the system and enterpriselevel, can take weeks. Our ongoing work focuses on a formal framework for the development of tools to specifically ensure that the engineer can make informed tradeoff decisions and that the evaluation is conducted on time.

The remainder of this paper is organized as follows: Section 2 describes the state of the art which focuses on understanding the procedures in the automotive industry for design optimization specifically focusing on sustainability attributes. In addition, we studied representative approaches for capturing the information in a typical CAE process. Section 3 focuses on development of the information model to formally capture the main components of information required in the CAE process with interfaces to using different sustainability metrics. The idea is to describe how data should be stored, linked, and accessed in the design process. These are represented in the form of class diagrams modeled using Unified Modeling Language (UML) [9] to describe the structure of the systems through classes and attributes and the relationships between the different classes. In Section 4, we discuss the definition, design, and implementation of a Proof-of-Concept prototype representative of the target assessment process. We shall illustrate the use of the information model defined in this project to facilitate two scenarios: current sequential CAE-LCA vs proposed SCAE. In Section 5, we analyze our Proof-ofConcept and establish the efficacy of our work in supporting the target assessment/CAE process for both traditional and sustainability targets. Section 6 concludes and summarizes ongoing and potential extensions of this approach for future activities.

\section{State of the art}

\subsection{State of the art in automotive CAE and design optimization}

The state of the art study focuses on understanding the different tools (in the automotive industry) that are used and the context (or attribute) to which they are applied and how the results are used. The objective is that our proposed SCAE framework should be aligned with the current approaches used within the industry.

CAE tools are currently used for design verification and synthesis. They provide the basis for optimization, six sigma design and trade off analysis. The primary sustainability attributes that are currently assessed using CAE tools include (along with typical software, for some): 
1. Noise Vibration and Harshness (NASTRAN [10])

2. Safety - Crash safety (RADIOSS [11])

3. Durability (NASTRAN, ADAMS [12])

4. Vehicle Dynamics (ADAMS)

5. Aerodynamics (POWERFLOW [13])

6. Vehicle Performance \& Fuel Economy (Programs such as the Simulink Corporate Vehicle Simulation Program (S-CVSP) developed at Ford Motor Company)

7. Cost

8. Weight

The objective of the CAE attribute simulation is to ensure that the product design meets the cascaded targets and this is enabled through the following steps:

a. developing an optimal design predicted from CAE results

b. assessing robustness of the design

c. quantifying and analyzing competing trade offs

Upon completion of the specific attribute development (also concurrently, if situation warrants), the design is evaluated for "cross-attribute" compatibility. This assessment is undertaken using multifunctional optimization software tools, such as Multi-function Optimization and Visualization Environment (MOVE) [14] and ISIGHT [15].

CAE analysis and optimization is undertaken using a number of alternative methodologies and protocols. Some of those are briefly described below:

- Discrete, Finite Element simulations: These simulations are conducted using models that are geometry-based, discretized and analyzed to replicate "real life" events. The key interaction is between product data and the product data management (PDM) system.

- Finite Difference simulations: These simulations are used for fluid flow and acoustic analysis, where the geometry forms the boundaries of the air-cavity. The air-cavity is discretized and these models can be very large.

- Conceptual model simulations: These simulations use simplified models that can represent both geometry and non-physical parameters to analyze verification events. They can represent physical spatial configurations (for vehicle dynamics using ADAMS) or energy management (for fuel economy using S-CSVP) or occupant motion (for vehicle safety using MADYMO [16]).

- Non-CAE simulations: Many analyses (what-if scenarios) are conducted using Microsoft (MS) Excel, MS SharePoint or MS Access databases. These focus on "business-driven attributes" like cost and weight, which tend to be scalar entities. The analysis for many of the new sustainability attributes would belong here.

\subsection{State of the art in CAE representation}

We studied several representations that are used or proposed for CAE and evaluated their suitability to integrate to CAD and LCA phases.

\subsubsection{ISO 10303 Application Protocol (AP) 209}

ISO 10303-209, Application Protocol (AP): Composite and Metallic Structural Analysis and Related Design [17], provides the data structures for the exchange of product information specifically in the design and analysis phases for composite and metallic structural analysis. A brief overview of the 
representation in the design and analysis is shown in Figure 3, reproduced from a dated publication as reference [18]. The boundary conditions can be captured and shapes in the finite element model can be mapped to part geometries and linked to the product structure. However, an important feature lacking in this representation (and other CAE representations) is the delivery of usable (for CAE) information (e.g., weight, loads) from the design to the analysis phase, i.e., capturing it explicitly in a Bill of Analysis. A Bill of Analysis represents the product configuration specifically for analysis. Some of the desired information is generally available in the product structure, yet it is not translated, and has to be recalculated every time the CAE analysis is run, typically, using the native CAE software. In studying this AP, we do not focus on studying any specific material type, but on only the representation of the Finite Element Analysis and the corresponding exchange between design and analysis domains as a typical representation within the international standard ISO 10303. In a more recent reference [19], the authors

propose a conceptual data architecture that can provide the technical basis for making tighter integration of spatial and functional design, analysis-driven design and, eventually, opportunistic analysis more pervasive.

\section{REMAINDER OF THIS PAGE IS BLANK}




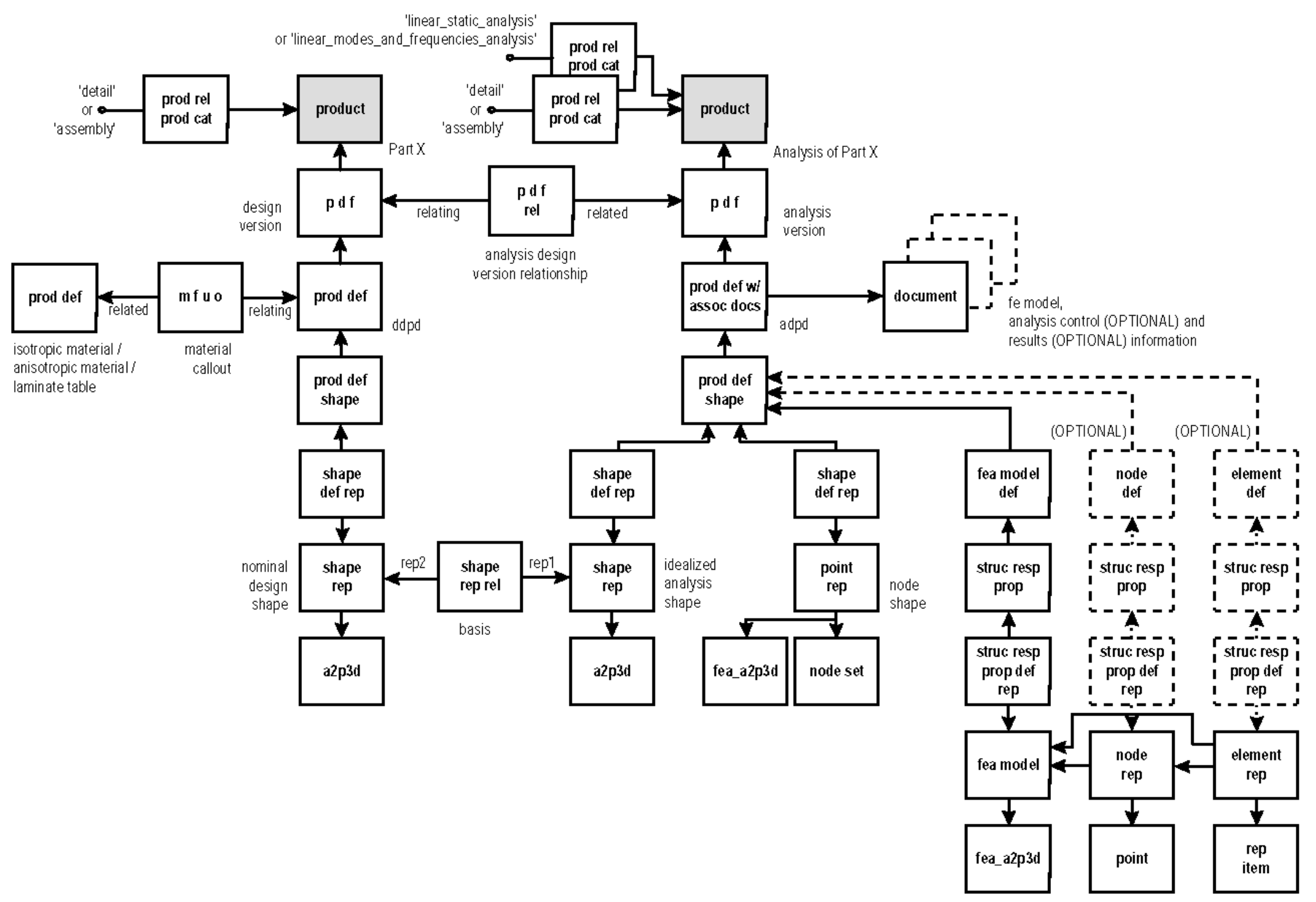

Figure 3: Analysis vs. Design Discipline Product Definition in AP 209. The "Analysis" is also a type of a "Product." The "Product" in the right hand side of the figure represents the analysis phase, while the "Product" in the Left Hand Side represents the design phase. Picture taken from [18]. 


\subsubsection{Commercial Software: Teamcenter}

To understand the commercial state of the art, we studied the Simulation/CAE data model implemented within the Siemens PLM's Teamcenter [20] solution suite; it is used at most automotive OEMs. It captures the relationship of CAE entities to Product entities, and a mechanism of transferring information (primarily geometry and associated material) from the product to the simulation/analysis application. Our extensive discussions with CAE-PLM Subject Matter Experts from automotive industry confirm that this representation faces paucity of content and lack of appropriate granularity. Much of the data stored are "file-based blobs" with closed-formats that cannot be extended to include newer sustainability-related attributes. The data is tightly embedded within proprietary material-related handlers and cannot be accessed externally, even with Application Programming Interfaces (APIs). The only way the data within the files can be accessed/processed is through solvers, i.e., the file has to be read into the specific application. The large number of CAE applications compounds the problem furthermore.

\subsubsection{Design - Analysis Integration Model proposed at NIST}

The Design-Analysis Integration Model (DAIM) is a conceptual data architecture for design-analysis integration [21]. The class diagram of the DAIM is shown in Figure 4. The Master Model serves as the global repository of information on a product being designed. Each FunctionalModel represents an abstraction of the product of interest to a specific functional/analysis domain. The two models are linked by two association classes. Idealization is the transformation that creates a functional/analysis model specific to a particular domain from the master model. Mapping is the reverse transformation of updating the master model based on results from the analysis domain.

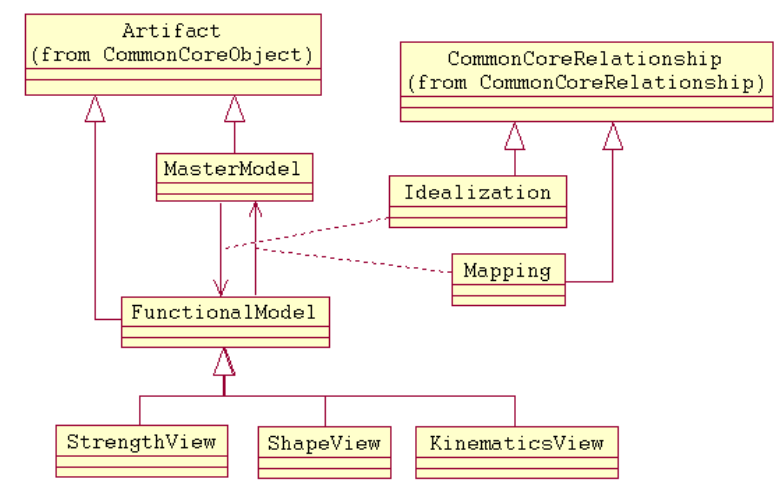

Figure 4: Class Diagram of the DAIM. Picture taken from [21]

\subsubsection{ASAM-ODS Model for Physical Testing}

Product verification is a critical portion of automotive development. Analytical testing (CAE) and physical testing are two parallel mechanisms to accomplish the objective. There is significant similarity in the process steps and the type of data that are managed in each process. As shown in Figure 5, the information elements within the two streams of verification mirror each other, and the standard industry practice is to compare elements from the two to ensure compliance and CAE correlation.

CAE information management

\begin{tabular}{|c|}
\hline Model development \\
\hline Simulation management \\
\hline CAE results management \\
\hline
\end{tabular}

Physical test information management

\begin{tabular}{|c|}
\hline Prototype build development \\
\hline Test data system \\
\hline Test results management \\
\hline
\end{tabular}

Figure 5: Similarity in process and type of data managed in analytical testing (CAE) and physical testing. 
Both CAE and testing support multiple customer requirements (safety, climate control, etc.), with multiple test stands (testing)/solvers (CAE) generating significant amount of similar results data that are processed using analysis tools to generate similar reports used to assess compliance to requirements.

While the processes and the data management requirements are similar for CAE and Test, the industry data model standards are not currently similar. There is no comprehensive standard for CAE but there exists one for Testing, which is called as ASAM-ODS.

The Association for Standardisation of Automation and Measuring systems (ASAM) [22] offers an infrastructure that allows vendors to develop software components in the area of computer aided test and measuring systems. The Open Data Service (ODS) [23] is described as "that part of the ASAM standard which is concerned with the storage and retrieval of information” and focuses strongly on physical testing. The overall model is shown in Figure 6.

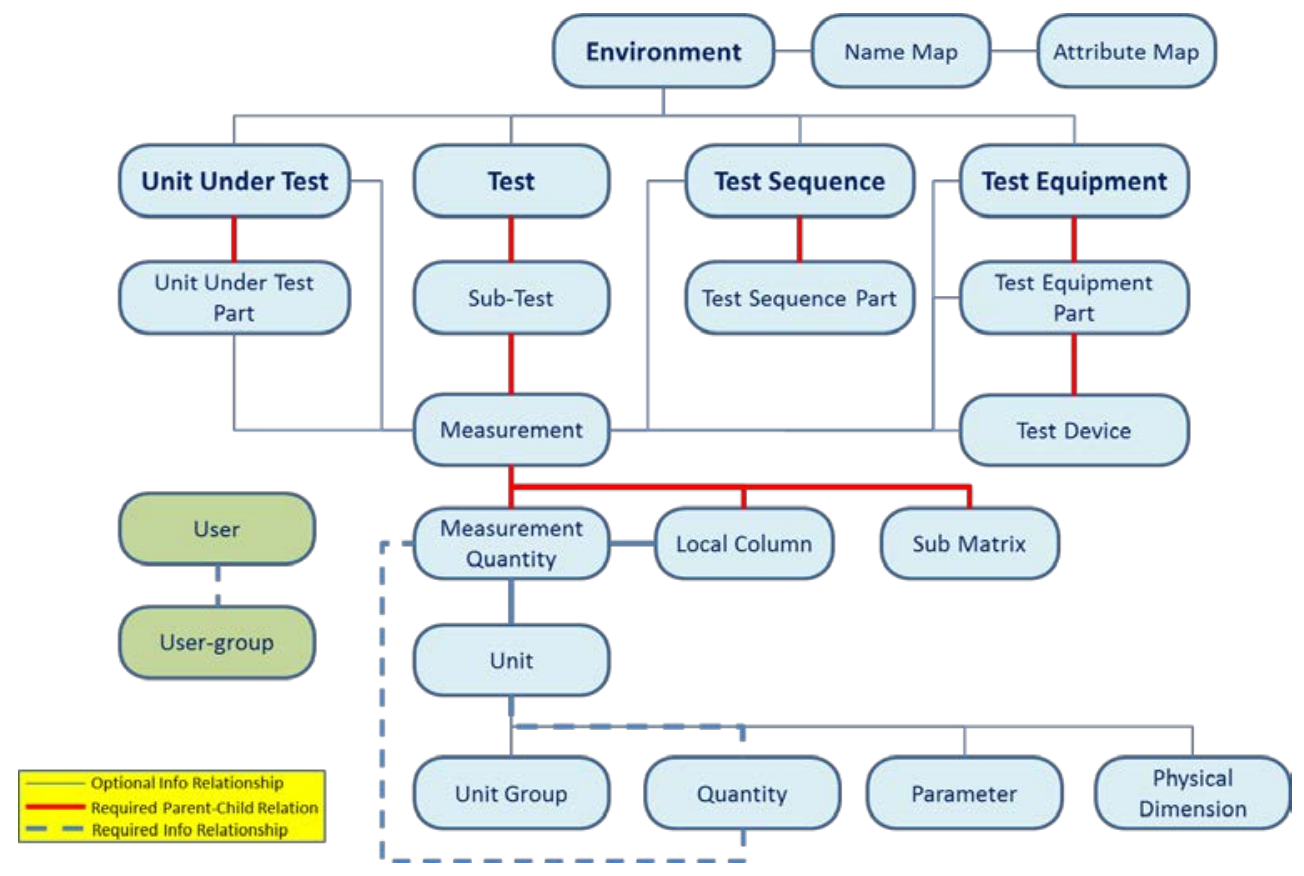

Figure 6: ASAM ODS model for physical testing. Figure adapted from [23] for ease of explanation.

We believe that the ASAM-ODS can serve as a basis for CAE data management along with the CPMDAIM described in the previous section because of its following properties:

1. comprehensiveness (supporting all customer requirements)

2. transportability (supports multiple testing hardware from multiple vendors)

3. broad acceptance of the testing standard in the automotive industry

4. inherent similarities of the business processes and the data structure/content/format between testing and $\mathrm{CAE}$

The proposed SCAE Information Model is described in the next section.

\section{Development of the information model}

To achieve effective design optimization for sustainability, we will need inputs from CAD, CAE, and LCA. In a previous project [7], we have developed information models to obtain information from the 
LCA and CAD domains. Those were used to integrate the CAD, CAE, and LCA information. The CAE information models were created as a part of this current project.

The class diagram of our proposed SCAE Information Model is shown in Figure 7. We based it on the CPM-DAIM model from NIST and the ASAM-ODS Model commonly used for physical tests. The ASAM-ODS model forms the basis of the core CAE representation, which includes concepts such as SCAEEnvironment, SCAEEquipment (solver) SCAEType, SCAESteps, SCAEResults. The CPM-DAIM forms the basis for exchange of information across the product design and analysis domains, i.e., through idealization and mapping operations. Information models from our previous work, whose requirements were mentioned in [7], are extended to enable the inclusion of CAE-specific information, such as idealizedMaterial, centerOfGravity, momentsOfInertia into the SCAE domain.

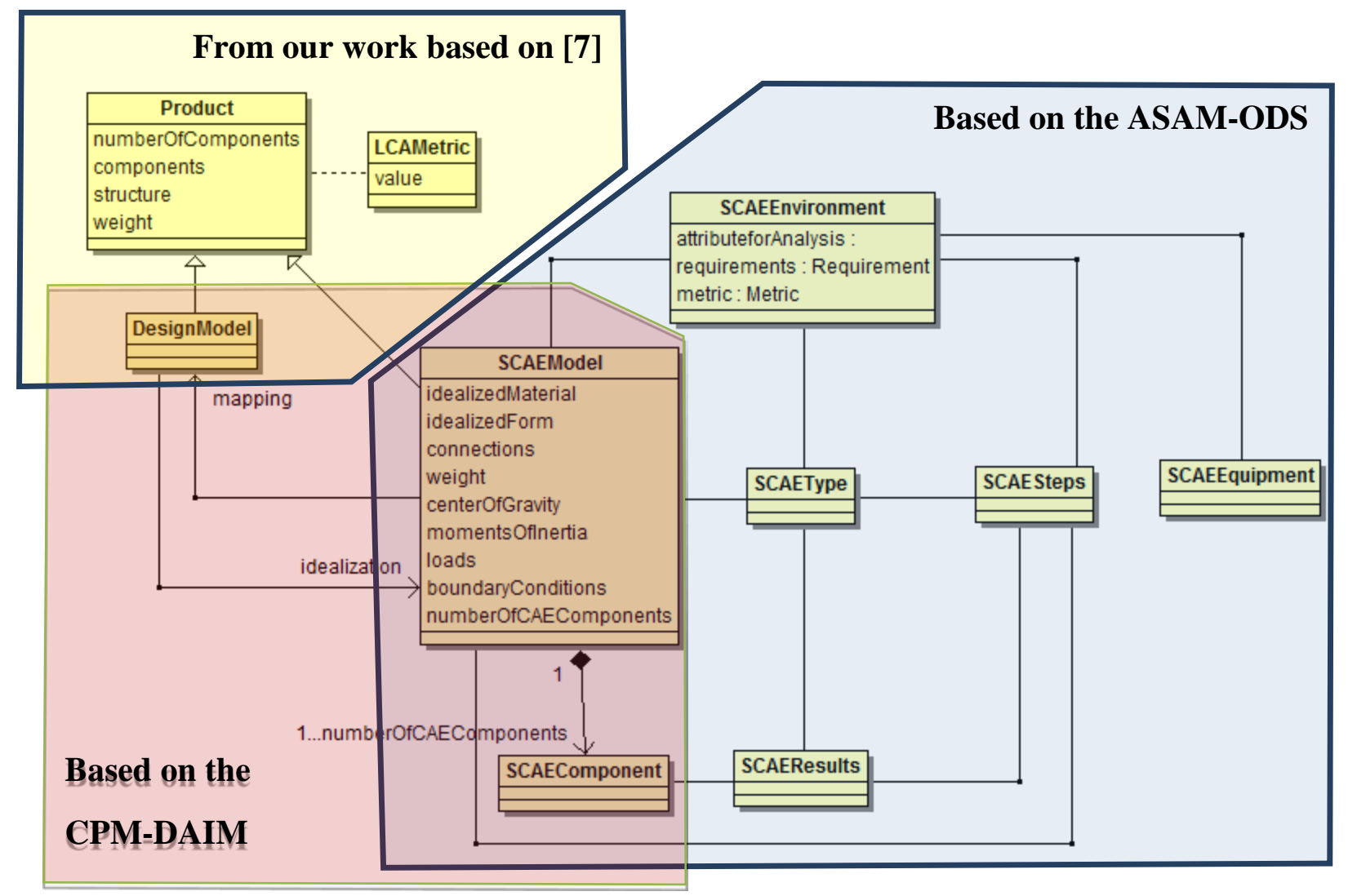

Figure 7: Proposed SCAE Information Model. The class names and connections are expected to be self-explanatory. The LCA data will be accessed through the "Product" class which was defined based on the work presented in [7].

We reiterate that one of the most important features lacking in current CAE representations is the absence of useful information (e.g., weight, boundary conditions, loads) in the Bill of Analysis (represented here by the CAEModel). This information has to be recalculated every time the CAE analysis is run, typically, using the native CAE software. Instead, we propose that in combination with the product and LCA models we have proposed in our own work that derives from [7], such information can be idealized and stored in the CAEModel. Further details of information constructs to be used depend on the nature of the attribute used for analysis, the type of the CAE analysis (linear, etc.), and the steps used in the CAE.

This information model was applied to a Proof of Concept scenario described in the next section. 


\section{Proof of Concept (PoC)}

In this section, we present how we defined the scope of the Proof of Concept, and some details on the implementation followed by the results.

\subsection{Identification of the scope of the PoC}

\subsubsection{The example product/assembly}

For this project, we have chosen a bumper assembly that is representative of a typical vehicle design environment. The example reflects the fact that, in a typical vehicle design environment, a designer designs on one or a small number of parts, not on a larger assembly or system. In Figure 8, the schematic of the bumper assembly is shown. It comprises three main components: the bumper beam, the absorbers (crushers), and the plates.

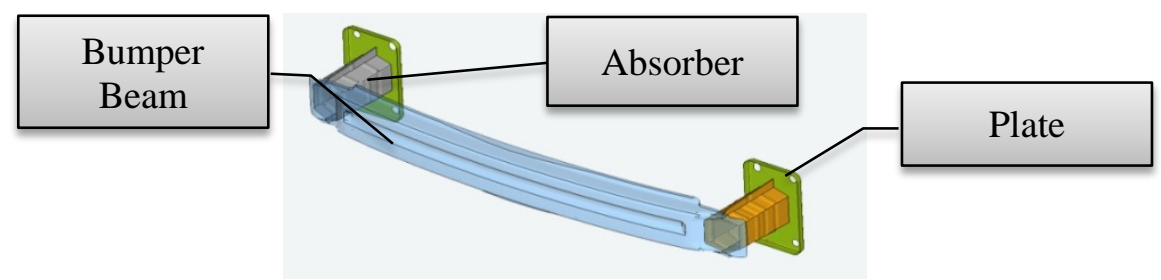

Figure 8: Schematic of a bumper assembly selected as example for the Proof of Concept

\subsubsection{Attributes for Cascading and analysis}

For this PoC, the intent was to choose attributes such that we can illustrate the current sequential process of Initial design -> CAE -> Feasible Design -> Sustainability Analysis and the proposed SCAE, which would provide Sustainability analysis upstream. This is shown with a simple schematic in Figure 9.

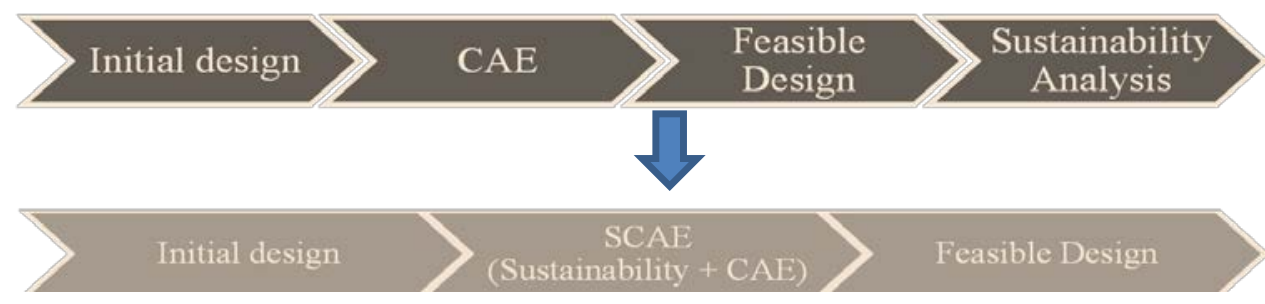

Figure 9: The purpose of the PoC is to demonstrate the paradigm change that incorporates sustainability analysis into the CAE phase so that the feasible design accounts for it.

Accordingly, we chose the following attributes:

- Cost: This is one of the simplest product attributes to understand, yet is also the most critical attribute in terms of driving decision making. Each BOM line has an associated cost, and these costs roll-up naturally from the lower levels to higher levels, providing an intuitive way of understanding the behavior. This is, in many cases, a decidedly business-oriented attribute, as opposed to a functionallyoriented attribute.

- Weight: Vehicle weight has increasingly become a very important attribute to manage in the vehicle design process. It acts as a proxy to a number of other performance related characteristics (e.g., safety, fuel economy), and, sometimes, business related attributes (e.g., cost) and sustainability attributes. Like cost, it is easily rolled up from lower levels to higher levels.

- Carbon Footprint: This is the chosen attribute for illustrating the incorporation of a sustainability attribute into the traditional vehicle design process. Since this is a well-explored aspect of 
sustainability, the use of this attribute in this PoC provides a clear illustration of the applicability of the SCAE information model. It should be noted that, as with any measurement and reporting system, there is uncertainty associated with the carbon footprint measurement process. In particular, in this work, we do not focus on choosing the right process. The objective is to demonstrate the feasibility of a system that has appropriate placeholders to obtain such information from various sources.

- Water consumption: Water consumption is being viewed as an extremely critical attribute of sustainability. It should be noted that, in public literature, the only data on water consumption that is available is the amount consumed during the production of the raw material.

- Performance attributes: Safety and Damageability are important performance attributes for bumper design. Under low speed impact, the bumper must not damage components, such as the radiator and the condenser. Bumper intrusion is defined as the difference of the displacement of the center of mass of the vehicle and a node at the inner edge of the bumper, and is usually restricted to the distance to the radiator. Safety, on the other hand, is evaluated on the basis of high speed impact; under high speed impact, the bumper must transmit just enough force to the airbag sensor so that it can be deployed. For this case, we will consider only the low speed impact and therefore, use "bumper intrusion" as the variable used to evaluate damageability.

\subsubsection{Setting targets}

The targets for the bumper assembly are manually set as shown in Figure 10. This PoC focuses exclusively on SCAE of the Bumper Beam. The Absorber is assumed to be carried over, i.e., used as is from the prior vehicle and, therefore, its values are fixed. Generally, a new vehicle is a revision of an existing product, which implies that many of the parts are carried over. Hence, the overall vehicle target is based on targets from the prior vehicle, with accommodations to new regulations or customer wants. This methodology was used to establish targets in this PoC also. Targets for Water and Carbon footprint for the Bumper Beam are manually decided as $3277 \mathrm{~L}$ of water and $590 \mathrm{~kg}$ of $\mathrm{CO}_{2}$ respectively after studying feasible ranges for the chosen Steel and Aluminum materials from the Granta Design Cambridge Engineering Selector software. The performance requirement is that the IIHS $9.66 \mathrm{~km} / \mathrm{h}(6 \mathrm{mph})$ centerline low-speed impact test produces a bumper intrusion should be less than $14 \mathrm{~cm}$. For the bumper beam, targets for weight (10.5 kg), cost (\$25) and the bumper intrusion are slight modifications of real targets (from confidentially view point).

\begin{tabular}{|c|c|c|c|c|c|}
\hline \multirow[t]{2}{*}{ TARGETS } & $\begin{array}{c}\text { Cost } \\
\text { (\$) }\end{array}$ & $\begin{array}{l}\text { Weight } \\
\text { (kg) }\end{array}$ & $\begin{array}{l}\text { Bumper Intrusion } \\
(\mathrm{cm})\end{array}$ & $\begin{array}{l}\text { Water } \\
\text { (L) }\end{array}$ & $\begin{array}{l}\text { Carbon Footprint } \\
\left(\mathrm{kg} \text { of } \mathrm{CO}_{2}\right)\end{array}$ \\
\hline & \multicolumn{5}{|c|}{--- } \\
\hline Bumper Beam & 25 & 10.5 & 14 & 3277 & 590 \\
\hline Absorber & \multicolumn{5}{|c|}{ Carry Over from previous year. } \\
\hline
\end{tabular}

Figure 10: Targets set for the bumper assembly. The PoC focuses mainly on the Bumper Beam. The Absorber is assumed to be a carryover and, therefore, its values are fixed. For the bumper beam, values for the bumper intrusion, weight and cost are modifications created from realistic targets. Values for Water and Carbon footprint targets are manually decided. 


\subsection{Implementation of the proof of concept}

For the Proof of Concept, we implemented a basic Excel-based solution.

The results for the CAE are obtained from the software ABAQUS/RADIOSS. The vehicle mass (not described in this paper) is assumed to be a point mass (not described in this paper) and it is assumed that the vehicle is constrained to move only along a longitudinal direction under the impact.

Values for sustainability metrics are obtained by calculations from the Granta Design Cambridge Engineering Selector software. It should be noted that our previously designed SIED Information Model serves the purpose of providing placeholders for the sustainability analyses very well. Therefore the SIED model has been reused, i.e., no new models to capture sustainability data were required.

\subsection{Documentation and analysis of results}

It should be noted that the numbers used in this PoC are not indicative of any vehicle program within any current automotive program. The targets and evaluation results are specific to this case.

\subsubsection{Scenario 1 (Current CAE-LCA Sequential process):}

This represents the current product development process and follows a sequential activity that starts with the initial product design, followed by CAE analyses in a recursive loop until a feasible product is obtained. Sustainability analyses are considered after-the-fact and are rarely considered for modifying the design.

Thus, in this scenario, we obtain the weights of the feasible design (that meets the required Safety and Damageability performance requirements) and then estimate the Water Consumption and Carbon Footprint. The results are shown in Figure 11.

\section{REMAINDER OF THIS PAGE IS BLANK}


STEP I: Use initial design to conduct CAE analysis and obtain feasible design with weight. This design, i.e., bumper beam made of steel UNS S20500 weighing $7.64 \mathrm{~kg}$ is then finalized

\begin{tabular}{|c|c|c|c|c|c|c|}
\hline & & $\begin{array}{c}\text { Cost } \\
(\$)\end{array}$ & $\begin{array}{l}\text { Weight } \\
\text { (kg) }\end{array}$ & $\begin{array}{c}\text { Bumper Intrusion } \\
\text { (cm) } \\
\end{array}$ & $\begin{array}{l}\text { Water } \\
\text { (L) }\end{array}$ & $\begin{array}{l}\text { Carbon Footprint } \\
\left(\mathrm{kg} \text { of } \mathrm{CO}_{2}\right)\end{array}$ \\
\hline \multicolumn{7}{|l|}{ Bumper assembly } \\
\hline & $\begin{array}{l}\text { Bumper Beam \#1 } \\
\text { (Steel UNS \$20500) }\end{array}$ & 21.63 & 7.64 & 11.9 & - & - \\
\hline & Absorber & \multicolumn{5}{|c|}{ Carry Over from previous year. } \\
\hline
\end{tabular}

STEP II: Use the finalized design to validate water consumption target. In this case, the targets are met. The value of water consumption is obtained from Granta Design's Cambridge Engineering Selector; it depends on the material (Steel UNS S20500) and its weight (7.64 kg).

\begin{tabular}{|c|c|c|c|c|c|c|}
\hline & & $\begin{array}{c}\text { Cost } \\
(\$)\end{array}$ & $\begin{array}{l}\text { Weight } \\
\text { (kg) }\end{array}$ & $\begin{array}{c}\text { Bumper Intrusion } \\
(\mathrm{cm})\end{array}$ & $\begin{array}{l}\text { Water } \\
\text { (L) }\end{array}$ & $\begin{array}{c}\text { Carbon Footprint } \\
\text { (kg of } \mathrm{CO} \text { ) }\end{array}$ \\
\hline \multicolumn{7}{|l|}{ Bumper assembly } \\
\hline & $\begin{array}{l}\text { Bumper Beam \#1 } \\
\text { (Steel UNS S20500) }\end{array}$ & 21.63 & 7.64 & 11.9 & 855.5 & \\
\hline & Absorber & \multicolumn{5}{|c|}{ Carry Over from previous year. } \\
\hline
\end{tabular}

STEP III: Use the finalized design to validate carbon footprint target. The carbon footprint value is obtained from Granta Design's Cambridge Engineering Selector and it depends on the material (Steel UNS S20500), its weight (7.64 kg), manufacturing process (rolling), use life (12 years), km driven per year (24k), fuel type consumed (gasoline), vehicle type (small sedan). In this case, the targets are not met, and a potential change to the design might have been required, but it would be too late to undertake that change.

\begin{tabular}{|c|c|c|c|c|c|c|}
\hline & & $\begin{array}{c}\text { Cost } \\
(\$)\end{array}$ & $\begin{array}{l}\text { Weight } \\
\text { (kg) }\end{array}$ & $\begin{array}{c}\text { Bumper Intrusion } \\
(\mathrm{cm})\end{array}$ & $\begin{array}{l}\text { Water } \\
\text { (L) }\end{array}$ & $\begin{array}{c}\text { Carbon Footprint } \\
\text { (kg of } \mathrm{CO} 2 \text { ) }\end{array}$ \\
\hline \multicolumn{7}{|l|}{ Bumper assembly } \\
\hline & $\begin{array}{l}\text { Bumper Beam \#1 } \\
\text { (Steel UNS S20500) }\end{array}$ & 21.63 & 7.64 & 11.9 & 855.5 & 647.3 \\
\hline & Absorber & \multicolumn{5}{|c|}{ arry Over from previous year. } \\
\hline
\end{tabular}

Figure 11: Results of the current sequential typical CAE followed by LCA process supported by the SCAE information model.

In this case, the carbon footprint target is not met, and a potential change to the design might have been required. Since it might be too late to update the design, the sustainability target values may have to be rebalanced and compensated elsewhere.

\subsubsection{Scenario 2 (SCAE - Holistic Process):}

We propose to include sustainability analysis into the CAE phase, such that an optimal design is obtained through the recursive design-analysis activity. Though we are not proposing any new CAE solver technology, we state that we can accomplish significant sustainability improvements through the simultaneous use of existing CAE and LCA tools. The goal of the PoC is to demonstrate this.

In this scenario (Figure 12), we obtain the weights of the feasible design following a simultaneous assessment of alternatives with values of the sustainability attributes. 


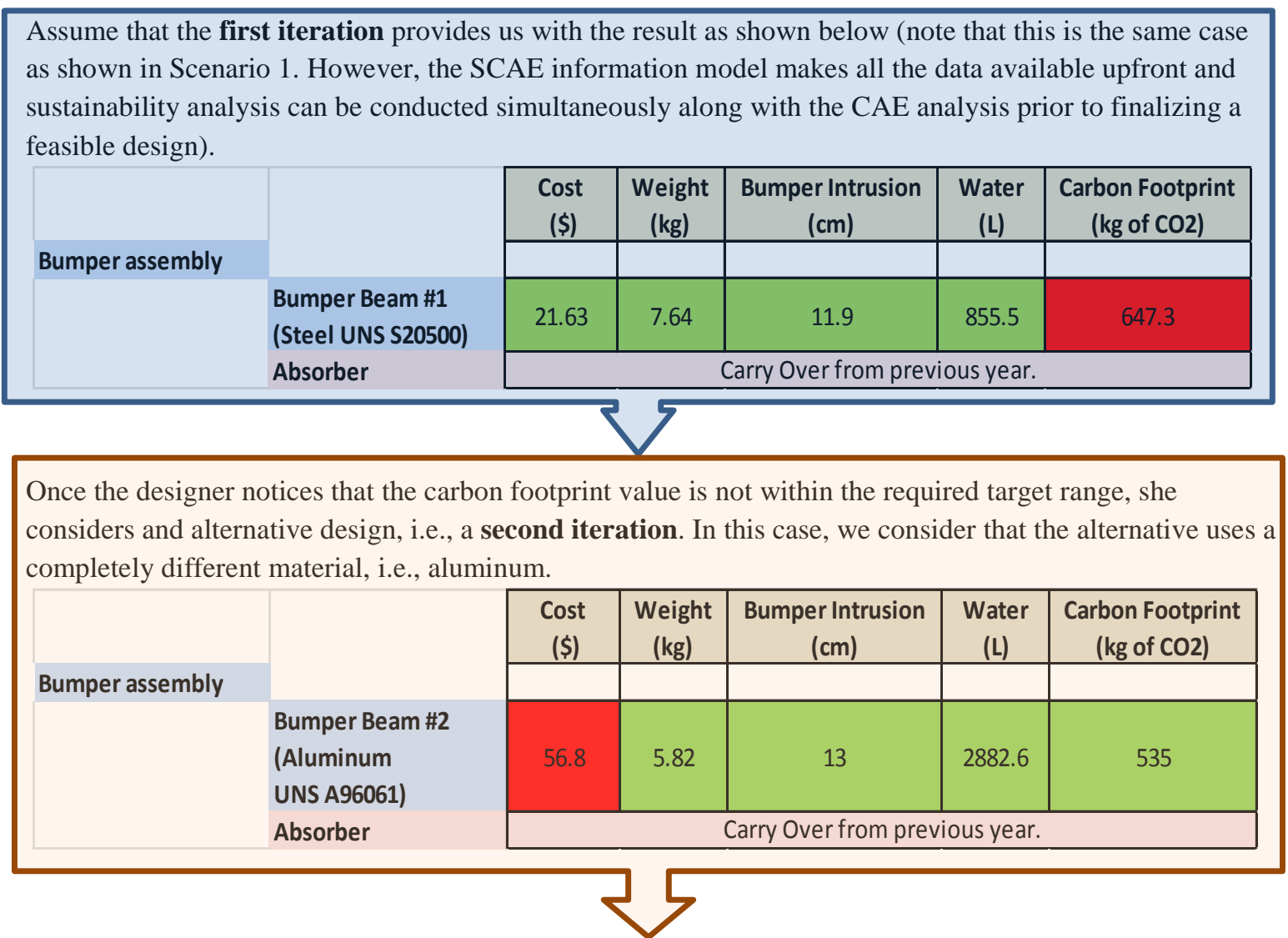

Figure 12: Results of the proposed SCAE (CAE and Sustainability analysis conducted simultaneously) process supported by the SCAE information model.

In this case, the Bumper Beam \#2 satisfies all the performance and sustainability criteria. It should be noted that while the carbon footprint of aluminum production and corresponding manufacturing process is significantly higher than that of steel, the use phase of the automobile and recyclability significantly impact the overall CF. In other words, a reduction of less than $2 \mathrm{~kg}$ in the weight of the bumper significantly impacts the carbon footprint of the vehicle. Also of note is the significant (about $300 \%$ ) increase in the water consumption due to the use of aluminum. While it is in the safe limits in this PoC, the manufacturer has to explain/address the tradeoff justified by such a large increase.

Note that to fully exploit the SCAE, it is possible to do a design change at the level of the geometry to meet the targets. However, typically, an engineer does not undertake major design changes, because the complex nature of an automobile implies that a design change will impact several systems and subsystems; that analysis and negotiation is avoided as much as possible. Furthermore, certain designs, such as the bumper, are driven by the styling and cannot be changed. As a result, the most common approach is to change the material of the product.

In this scenario, for the second iteration, the designer is faced with the problem that her cost target is not met. Normal industry practice would lead companies to make cost-driven decisions and the significant cost penalty could be a negative deterrence.

Such situations are common and typical in the automotive sector. Obtaining a unique optimal solution that satisfies all the requirements is almost never possible. So, the designer has to evaluate multiple 
alternatives and conduct trade-offs by relaxing one or more targets, i.e., conduct target rebalancing before finalizing the design.

\section{Discussion}

\subsection{Benefits of the PoC}

The efficacy of the proposed SCAE information model, as evaluated from the Proof of Concept is summarized in Figure 13 and discussed in brief in this section.

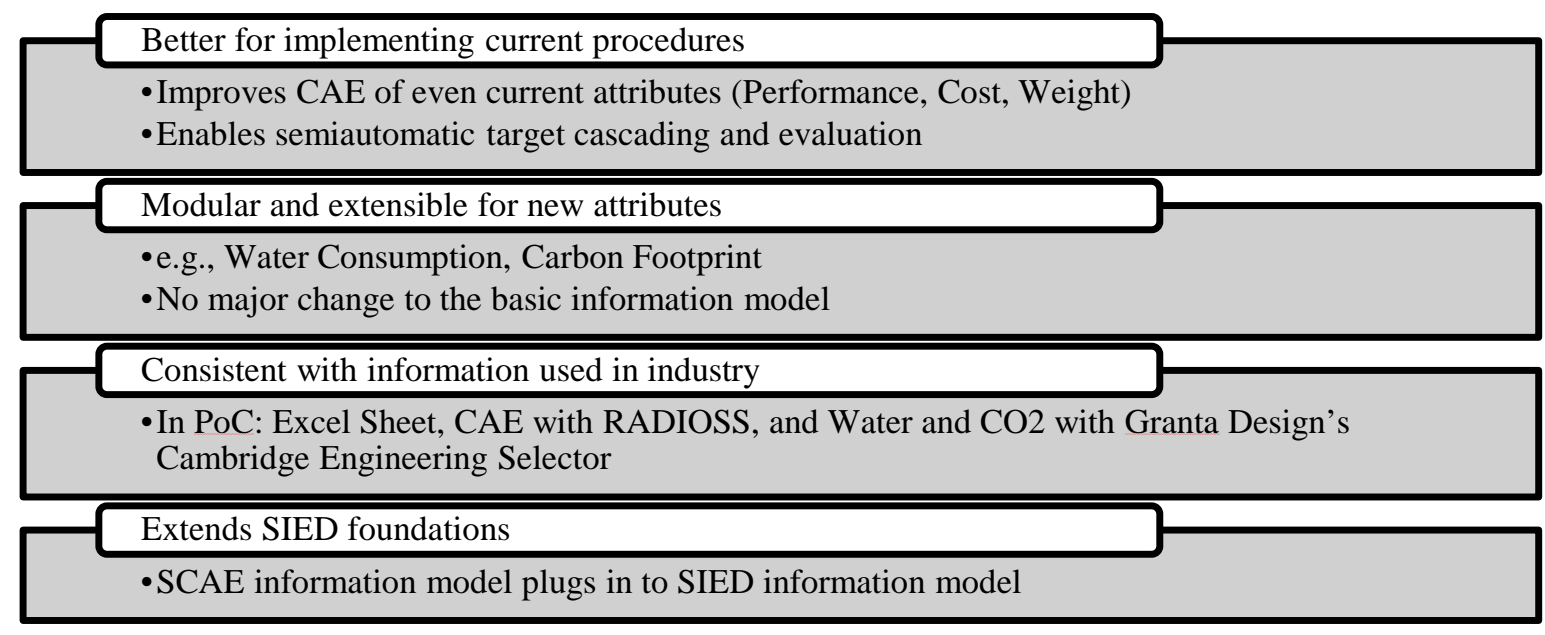

Figure 13: Summary of the benefits of the SCAE model demonstrated through the PoC

1. Applicability in better implementation of current procedures: We can use the information model to improve CAE analysis of even those attributes, e.g., cost and weight that are currently managed by the industry. Currently, such an information model that has consistent placeholders to capture even basic cost and weight attributes in an integrated manner with CAE from across the lifecycle is unavailable. The Proof of Concept shows that once the information is appropriately made available (detailed information model is not shown in this paper), it enables the crucial process of target cascading and target evaluation to be conducted semi-automatically.

2. Modularity and Extensibility to incorporate new attributes: Incorporation of new attributes, such as water consumption or carbon footprint, does not require a major change to the information model or the procedure that was used for existing attributes, such as Cost and Weight.

3. Consistency of instances with information model: The information constructs used in the Proof of Concept implementation (Excel, CAE with RADIOSS, and water consumption and carbon footprint with Granta Design’s Cambridge Engineering Selector) are consistent with the Classes developed earlier in this work and SIED information model based on [7].

To illustrate this, we present the instance diagram (Figure 14) of just the first part of the Scenario 1 described earlier, i.e., traditional CAE for Damageability using Bumper Intrusion as the variable for measurement. 


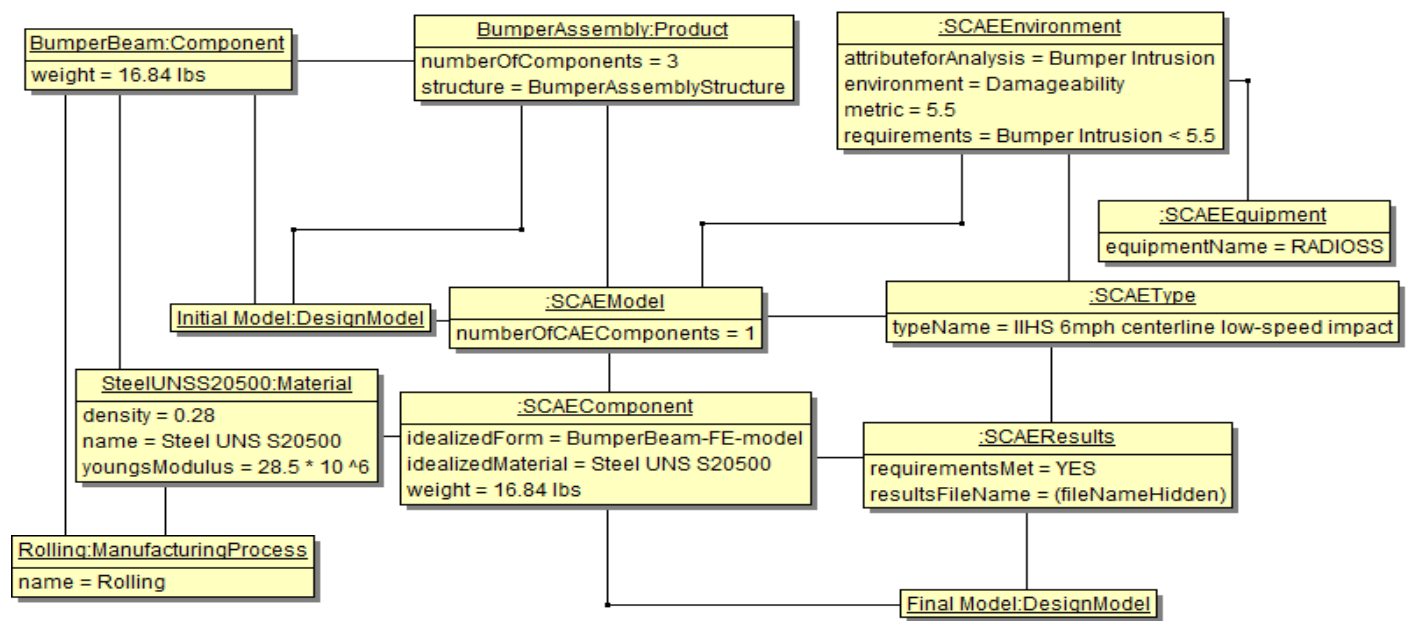

Figure 14: Instance Diagram for traditional CAE derived from Classes created for SCAE and SIED. (Only some of the properties and linkages are shown to maintain readability of the picture.)

After implementing the SCAE Proof-of-Concept, the instance diagram for SCAE of the Bumper Beam using steel design is as shown in Figure 15. Note that the figure partially details the SCAE evaluation of carbon footprint only. The instance diagram for damageability is similar to the one in Figure 15. The instance diagram for water consumption is not shown. The results of this SCAE for the Steel design are input to create the "feasible model." A similar SCAE for the aluminum design (described earlier) is a necessary input (not shown in Figure 15).

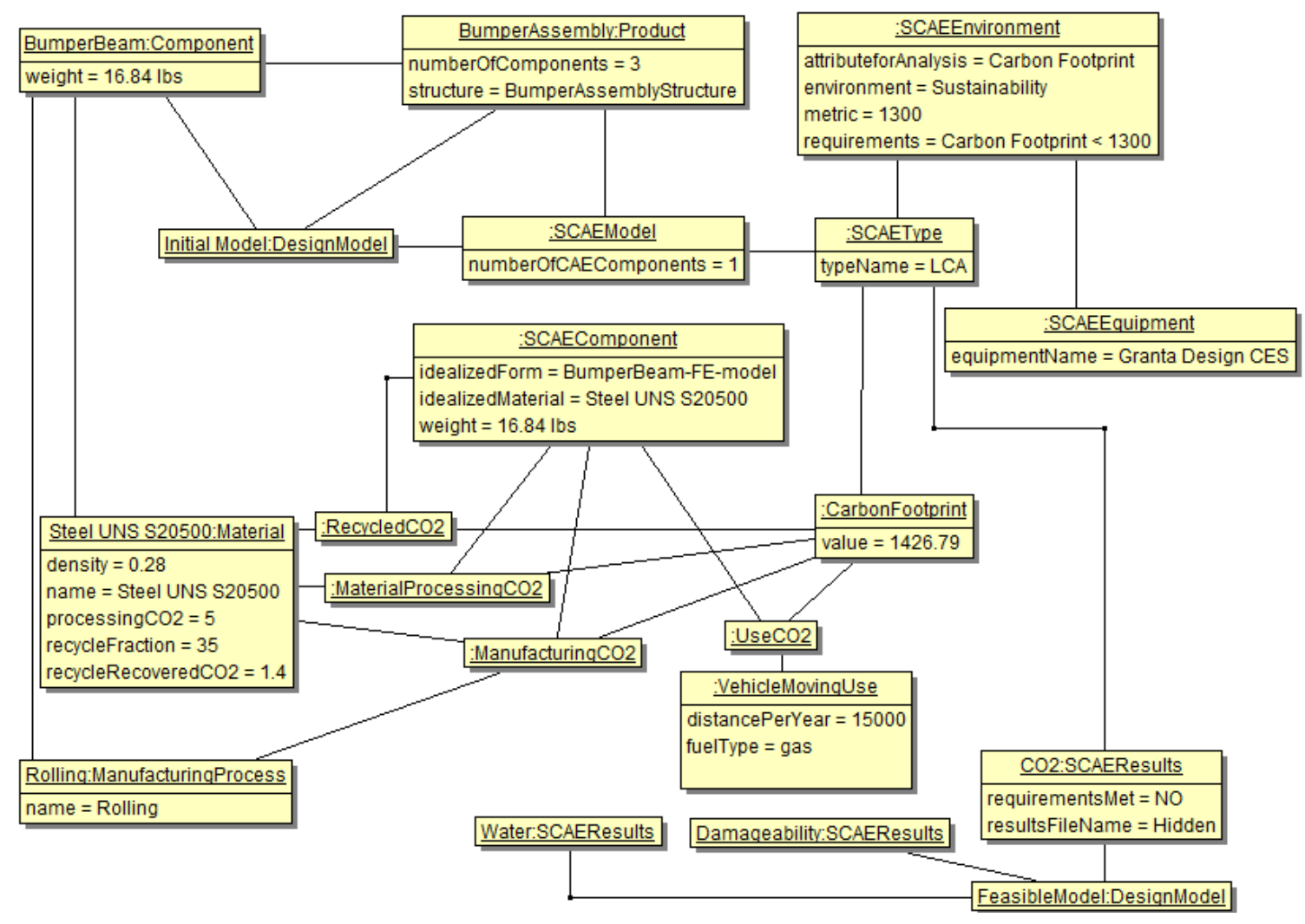

Figure 15: Instance Diagram for SCAE of the Steel-based design for the Bumper Beam derived from classes created for SCAE and SIED. (Only some of the properties and linkages are shown to maintain readability of the picture.) 
4. Extensions to the SIED model: The SCAE model, proposed in this paper, plugs into the SIED information model that is based on [7]. The SIED UML model was only imported into the SCAE model and thus, reused. This enables us to ensure that the two projects are aligned and can be integrated to provide a holistic solution.

\subsection{Limitations of the Proof of Concept}

In determining the scope of the PoC, and its implementation approach, a number of decisions were made to ensure that it fits within the parameters of a feasibility study. In this section, we explicitly describe some of the key limitations, and also discuss potential roadmap to advance this implementation towards a production-ready solution.

- No formal software development process: Since the main intent of this exercise is to illustrate the concepts developed in this paper, the PoC development is limited to a bare-minimum approach. In particular, the normal artifacts that are deliverables of a standard software development process, such as requirements, use cases, test plans, are absent in this PoC. In transitioning from this stage to a production-ready application, the development of this solution must follow a rigorous Software Development process.

- The user interface is neither complete nor friendly: This component of the PoC study clearly needs an overhaul in its progression towards a production application. We chose to use Excel as the user-interface and manual export/import processes to minimize the resources spent on userinterface aspects. In a production scenario, we will not have that choice.

\section{Conclusion and future work}

The purpose of the effort discussed in this paper was to develop the framework called Sustainability Computer Aided Engineering (SCAE) and determine its feasibility for industrial applications. The premise of the work is the fact that the lack of standard and consistent information models that captures relevant information across the entire lifecycle affects the target management process in product development. Important work done is as follows:

1. Development of information model: Formalization of the main components of information required in the CAE process with interfaces to using different sustainability metrics.

2. Proof of Concept (PoC) prototype: Definition, design, and implementation of a prototype representative of the target assessment process with the primary goal of illustrating the use of the information model defined this project to facilitate two scenarios: current sequential CAE-LCA vs proposed SCAE.

3. Analysis of the PoC: Briefly establishing, in the context of the PoC, the efficacy of the proposed information model supporting the target assessment/CAE process for both traditional and sustainability targets).

\subsection{Estimates of technical feasibility}

Based on our analytical investigations, in-depth knowledge of automotive product creation process, and supporting insights gained via the Proof-of-Concept implementation, we estimate that the technical feasibility of the approach proposed in this paper is excellent, with near $100 \%$ certainty of translating into the Sustainability Integrated into Early Design (SCAE) commercial product, in particular the integration of SCAE with ongoing work on Sustainability Integrated into Early Design (SIED). 
The work presented in this paper aligns well with real-life automotive product creation processes. Currently, such an information model that has consistent placeholders to enable target evaluation by capturing even basic cost and weight attributes from across the lifecycle is unavailable. Leveraging the depth of knowledge available with the core team and their contacts in the industry, we strived to tailor our approach to achieve a close alignment with current practices in the automotive industry. Our expectation is that this alignment will almost certainly guarantee a quick uptake and transition to the new tool. At the same time, we have structured the implementation such that future improvements in the approach can be added to it in an incremental and modular manner. This avenue, in our opinion, eliminates most risks associated with the technical feasibility of implementation.

\subsection{Potential research directions}

In addition to a number of actions that can be taken to move the PoC implementation to a productionready solution, there are several key areas that deserve further research and development and subsequent inclusion in the production solution. We describe three such important opportunities here that is the basis of our ongoing research.

1. Assessing the meaning of the status of the targets: Up until now, the work focuses on providing the status of the targets. However, currently, there are no tools to help the decision-maker make sense of the numbers, which will help her close the loop and make decisions that will impact target rebalancing, i.e., manage the tradeoffs due to deviations from the targets. In this regard, deviation value (status vs target) has some semantics. For example, a $2 \%$ cost deviation in a higher-end Shelby Mustang has completely different implications as compared to a $2 \%$ cost deviation on a Ford F-150. Furthermore, the designer needs to understand how the tradeoffs can be quantified, i.e., what is the tradeoff if the $\$ 2$ cost deviation is allowed to go through? This requires an approach to understand the interrelationships between the different values.

2. Timely evaluation of the status of the target: Note that in this section, we describe the concepts using carbon footprint as a representative attribute for sustainability. Similar issues apply for other sustainability attributes.

The engineer cannot make effective decisions due to the lack of timely evaluations of the status of the attribute across different phases of product development. Primary challenges are as follows:

a. Prohibitively long time taken to evaluate target status: Using a detailed LCA-based approach in calculating the carbon footprint of a vehicle during the early phases can take a prohibitively long time. Several times, CAE-like analyses take weeks to return a result and product development cannot be withheld for that long. If not completed on time, the evaluation is of no use in the tradeoff/target rebalancing analyses discussed previously.

b. Lack of detailed information in early phases: Typically, detailed information from across several phases of the lifecycle is required to conduct a sustainability analysis. Recent tools, such as AutoCAD Inventor and Solidworks, integrate computer aided design (CAD) systems with carbon footprint evaluators, e.g., Granta Design's Cambridge Engineering Selector, to yield carbon footprint estimates when embodiments and manufacturing information are provided. However, such detailed information is not available in the early phases of product design, much less integrated with it.

c. Inability to handle enterprise-level parameters in the evaluation: Product design and manufacturing in a large OEM depends on a very large number of enterprise-level parameters, e.g., supplier location and commonality of part, that should impact the calculation 
of the carbon footprint and are usually not incorporated in the normal calculations that limit themselves to a single product.

d. Inability to integrate and reuse different models to enable a systems level analysis: Several models capturing diverse views of different attributes need to be used concurrently within an enterprise. These interdependent models need to be integrated for effective systemlevel decision-making. In addition, model reuse is very much desired, but is carried out in an ad hoc manner.

Thus, there is a need for a rigorous framework to analyze/predict product sustainability virtually with incomplete information and comprehensive enterprise-level parameters. A model-based engineering (MBE) approach is expected to provide the ability to address the above-mentioned challenges. As a result, there is a significant industrial push toward the development of frameworks and information models compatible with MBE.

3. Incorporating manufacturing materials information into the upstream design phase - Currently, manufacturing process information, including materials that are used or consumed during the manufacturing is rarely available to the designer. This is very significant for evaluation of sustainability, since those materials that are consumed or wasted might be significant contributors to the overall sustainability. There is a need to integrate the "process material" information with the "product material" information. This includes the need to formalize the concept of a "Bill of Substance" and its integration with the Bill of Material. It is this kind of work that will lay the foundation for the second step toward Product Sustainability Management (PSM).

\section{Acknowledgment}

This material is based upon work funded, in part or service, by the National Institute of Standards and Technology under Order Number SB1341-11-SE-0873. Any opinions, findings, and conclusions or recommendations expressed in this publication are those of the authors and do not necessarily reflect the views of the National Institute of Standards and Technology.

We acknowledge the following Ford Motor Company personnel: Nand Kochhar, Chief Engineer and Executive Technical Leader of Global CAE, Laxmi Sivashankar, Manager of Global C3P Methods and System Integration Solutions, and Carrie Majeske, Product Sustainability Manager, for their multiple interactions, insights on what works, and the relevance and validity of our work (including the proof-ofconcept).

\section{Disclaimer}

No approval or endorsement of any commercial product by NIST is intended or implied. Certain commercial software are identified in this report to facilitate better understanding. Such identification does not imply recommendations or endorsement by NIST nor does it imply the software identified are necessarily the best available for the purpose.

\section{References}

[1] S. Rachuri, "Understanding sustainability of products," presented at the Indo-US Workshop on Designing Sustainable Products, Services, and Manufacturing Systems, Indian Institute of Science, Bangalore, India, 2009. 
[2] C. Jackson and D. Houlihan, “Greening Today’s Products: Sustainable Design Meets Engineering Innovation,” Aberdeen Group, 2008.

[3] “American Recovery and Reinvestment Act of 2009,” American Recovery and Reinvestment Act of 2009, 2009. http://www.recovery.gov.

[4] Organisation for Economic Co-operation and Development (OECD), "Conducting sustainability assessments,” OECD, Paris, France, 2008.

[5] International Organization for Standardization (ISO), "ISO 14040:2006 - Environmental Management - Life Cycle Assessment - Principles and framework,” 2006.

[6] W.-P. Schmidt and A. Taylor, "Ford of Europe's Product Sustainability Index," in Proceedings of the 13th CIRP International Conference on Life Cycle Engineering, Leuven, Switzerland, 2006.

[7] L. Patil, L. Srinivas, K. Murthy, D. Dutta, and R. Sudarsan, "Requirements for the development of a decision-support solution for sustainability target cascading,” in CASE'11, 2011, pp. 456-461.

[8] J. Lippert and K. Naughton, "Mulally Gives Ford Lightness Lead After Threat to End Explorer," Bloomberg Markets Magazine, Feb-2011.

[9] Object Management Group (OMG), “Unified Modeling Language,” 2011. http://www.uml.org/.

[10] “MSC Software Nastran,” 2012. http://www.mscsoftware.com/Products/CAE-Tools/MSCNastran.aspx.

[11] “Altair RADIOSS,” 2012. http://www.altairhyperworks.com/Product,51,RADIOSS.aspx.

[12] “MSC Software Adams," 2012. http://www.mscsoftware.com/Products/CAE-Tools/Adams.aspx.

[13] “Exa Powerflow,” 2012. http://www.exa.com/powerflow.html.

[14] K. Longacre, J. Vance, and R. Devries, "A Computer Tool to Facilitate Cross- Attribute Optimization," in 6th AIAA Symposium on Multidisciplinary Analysis and Optimization, Bellevue, WA, 1996, pp. 1275-1279.

[15] "Isight and the SIMULIA Execution Engine," 2012. http://www.3ds.com/products/simulia/ portfolio/isight-simulia-execution-engine/overview/.

[16] “MADYMO Product Suite,” 2012. http://www.tass-safe.com/en/products/madymo/.

[17] International Organization for Standardization (ISO), "ISO 10303-209:2001 - Industrial automation systems and integration - Product data representation and exchange - Part 209: Application protocol: Composite and metallic structural analysis and related design," 2001.

[18] PDES, Inc., “Recommended Practices for AP 209,” ME007.01.00, Jun. 1999.

[19] S. Fenves, Y. Choi, B. Gurumoorthy, G. Mocko, and R. Sriram, "Master Product Model for the Support of Tighter Integration of Spatial and Functional Design," National Institute of Standards and Technology (NIST), Technical Report NISTIR 7004, 2003.

[20] “Siemens PLM Teamcenter," 2012. http://www.plm.automation.siemens.com/en_us/ products/teamcenter/.

[21] S. Rachuri, M. Baysal, U. Roy, S. Foufou, C. Bock, S. Fenves, E. Subrahmanian, K. Lyons, and R. Sriram, "Information models for product representation: Core and assembly models," International Journal of Product Development, vol. 2, no. 3, pp. 207-235, 2005.

[22] "Association for Standardisation of Automation and Measuring Systems," 2012. http://www.asam.net.

[23] “ASAM ODS,” 2012. http://www.asam.net/nc/home/standards/standard-detail.html ?tx_rbwbmasamstandards_pi1\%5BshowUid\%5D=432. 